\title{
A TRANSFER PRINCIPLE FOR SIMPLE PROPERTIES OF THEORIES
}

\author{
MARK E. NADEL
}

\begin{abstract}
A notion of simple property of theories is introduced and it is shown that if $\mathrm{P}$ is a simple property of theories, $A$ countable admissible, and $M$ a structure in $A$, then $\mathrm{Th}_{A}(M)$ has property $\mathrm{P}$ iff $\mathrm{Th}_{\infty \omega}(M)$ has property P.
\end{abstract}

In our earlier paper [3] we showed that a model is $\equiv_{\infty}$ to an uncountable model iff there is an uncountable model of its complete theory with respect to some admissible set containing a copy of the given model. As we remarked there, though this result seemed very much like the well-known result of Gregory [1] and Ressayre [5], it was not clear if and how the former could be derived from the latter. It was only by examining Ressayre's proof that we were led to a proof of our result.

Subsequently, we observed that a number of results of a related character could be obtained. This in turn led us to formulate a "transfer principle." Roughly speaking, given a theorem of a type to be specified later which tells when a $\boldsymbol{\Sigma}$-definable theory on a countable set has some property $\mathrm{P}$, the "transfer principle" yields the theorem:

Let $A$ be countable admissible and let $M$ be a model in $A$. Then $\operatorname{Th}_{A}(M)$ has property $\mathrm{P}$ iff $\mathrm{Th}_{\infty}(M)$ has property $\mathrm{P}$.

Each of the results alluded to above is seen to be an instance of this "transfer principle." Nevertheless, we feel that our proof in [3] is still of interest since a much more direct argument is used in place of the GregoryRessayre theorem which would constitute the starting point for the application of the "transfer principle."

For the most part, it makes no difference whether or not the admissible sets considered are allowed to contain urelements. For this reason we suppress all mention of urelements until the very end where we make specific use of them. Consequently, we generally use $x^{+}$to denote the smallest admissible set containing $x$, and reserve the notation $\operatorname{HYP}(x)$ for emphasis in the use of urelements.

Given a structure $M$ and a fragment $L_{A}$, we use $\operatorname{Th}_{A}(M)$ to denote the set of all sentences of $L_{A}$ true in $M$. Similarly, we use $\operatorname{Th}_{\infty \omega \omega}(M)$ to denote the class of all sentences of $L_{\infty \omega}$ true in $M$.

If $A$ is admissible, then by a $\Sigma_{A}$ theory in $L_{A}$ we mean a class of sentences of $L_{A}$ definable over $A$ by a $\Sigma$ formula of set theory with parameters from $A$.

Received by the editors May 12, 1975.

AMS (MOS) subject classifications (1970). Primary 02H10; Secondary $02 \mathrm{H} 05$.

(๑) American Mathematical Society 1976 
Following our practice in [3], we use the notion of $\boldsymbol{\Sigma}_{A}$-saturated in the weak sense; namely $M$ is said to be a $\Sigma_{A}$-saturated model of a theory $T$ provided that there is some countable admissible $B \supseteq A$ with the same ordinals as $A$ which contains an isomorphic copy of $M$.

We will need the following results which were also basic for [3].

Lemma 1. Let $A$ be countable admissible and let $T$ be $a \boldsymbol{\Sigma}_{A}$ theory which has a model. Then $T$ has a $\boldsymbol{\Sigma}_{A}$-saturated model.

Lemma 2. Suppose $A$ is countable admissible and $M, N \in A$. Then $M \equiv_{A} N$ iff $M \cong N$.

Lemma 3. Let $A$ be countable admissible and $T$ a complete theory in $L_{A}$. If $T$ has a model in $A$, then $T$ has a unique $\boldsymbol{\Sigma}_{A}$-saturated model up to isomorphism.

We wish to thank S. Feferman and J. Stavi for their patience with an earlier version of these results.

1. For convenience we fix some $L$ and $L^{\prime}$. All admissible sets considered will contain $L$ and $L^{\prime}$. By an assignment $T^{\prime}$ of $\boldsymbol{\Sigma}$ theories in $L^{\prime}$ we mean a mapping which assigns to each countable admissible set $A$ a $\Sigma_{A}$ theory $T_{A}^{\prime}$ in $L_{A}^{\prime}$ such that if $B$ is also countable admissible and $A \subseteq B$, then $T_{A}^{\prime} \subseteq T_{B}^{\prime}$.

A property $\mathrm{P}$ is said to be a property of theories provided that if any theory $\Gamma$ has property $\mathbf{P}$ and $\Gamma^{\prime}$ is a theory logically equivalent to $\Gamma$, then $\Gamma^{\prime}$ has property $P$.

Definition 1. A property $\mathrm{P}$ of theories in $L_{\infty \omega}$ is said to be simple iff there is an assignment of $\boldsymbol{\Sigma}$ theories of $L^{\prime}, T^{\prime}$, such that for any countable admissible set $A$ :

(i) If $T$ is a theory in $L_{A}$ set primitive recursively definable on $A$, then $T$ has property $\mathrm{P}$ iff $T \cup T_{A}^{\prime}$ has a model; and

(ii) If $M$ is a structure for $L$ and $M$ has an expansion to a structure for $L \cup L^{\prime}$ satisfying $T_{M^{+}}^{\prime}$, then $M$ has an expansion to a structure for $L \cup L^{\prime}$ satisfying $T_{M^{++}}^{\prime}$.

Once this definition has been formulated, the desired theorem and its proof are easily found.

THEOREM 1. Let $\mathrm{P}$ be a simple property of theories. Let $A$ be a countable admissible set with $M \in A$ a structure for $L$. Then $\mathrm{Th}_{A}(M)$ has property $\mathrm{P}$ iff $\mathrm{Th}_{\infty \omega}(M)$ has property $\mathrm{P}$.

Proof. We must first show that with $A$ and $M$ as above, if $\operatorname{Th}_{A}(M)$ has property $\mathrm{P}$, then so does $\mathrm{Th}_{\infty \omega}(M)$. Let us therefore assume that $\mathrm{Th}_{A}(M)$ has property $\mathrm{P}$ and that $T_{A}^{\prime}$ is a $\boldsymbol{\Sigma}$-definable theory associated with $\mathrm{P}$ as in Definition 1.

Since $M \in A, \mathrm{Th}_{A}(M)$ is set primitively recursively definable on $A$, whence the $\Sigma_{A}$ theory $\operatorname{Th}_{A}(M) \cup T_{A}^{\prime}$ has a model. By Lemma 1 above, $\mathrm{Th}_{A}(M) \cup T_{A}^{\prime}$ has a $\Sigma_{A}$-saturated model $M^{*}$. Then, since $M^{*} \uparrow L \models$ $\mathrm{Th}_{A}(M)$ and $M^{*} \uparrow L$ is $\Sigma_{A}$-saturated (with respect to $L$ ), by Lemma 3 above, $M^{*} \uparrow L \cong M$. 
In particular then, $M$ has an expansion to a model of $T_{A}^{\prime}$, and since $M^{+} \subseteq A$, a fortiori, to a model of $T_{M^{+}}^{\prime}$. Hence, by (ii) of Definition $1, M$ has an expansion $N^{*}$ to a model of $T_{M^{++}}^{\prime}$. We now use the fact that within $M^{++}$ lies a Scott sentence $\sigma$ of $M$, characterizing $M$ up to $\equiv_{\infty \omega}$ (cf. [2]).

Now, since $N^{*} \vDash\{\sigma\} \cup T_{M^{++}}^{\prime}$ and $\{\sigma\}$ is set primitively recursively definable on $M^{++}$, by (i) of Definition 1, it follows that $\{\sigma\}$ has property $\mathrm{P}$. Finally since $\{\sigma\}$ and $\mathrm{Th}_{\infty \omega}(M)$ are equivalent, $\mathrm{Th}_{\infty \omega}(M)$ has property $\mathrm{P}$.

The converse is easy. Suppose $\operatorname{Th}_{\infty \omega}(M)$ has property $\mathrm{P}$. Then since $\mathrm{P}$ is a property of theories and $M$ has a Scott sentence in $A^{+}, \mathrm{Th}_{A^{+}}(M)$ has property P. Whence $\mathrm{Th}_{A^{+}}(M) \cup T_{A^{+}}^{\prime}$ has a model. Now, since $T_{A}^{\prime} \subseteq T_{A^{+}}^{\prime}$, $\mathrm{Th}_{A}(M) \cup T_{A}^{\prime}$ has a model, and so $\mathrm{Th}_{A}(M)$ has property $\mathrm{P}$.

2. We now give two applications of Theorem 1 .

Application 1. We show that if $A$ is countable admissible and $M \in A$, then $\mathrm{Th}_{A}(M)$ has an uncountable model iff $\mathrm{Th}_{\infty \mathrm{w}}(M)$ has an uncountable model.

By Theorem 1 it is sufficient to show that "-has an uncountable model" is a simple property of theories. First, it is obvious that "- has an uncountable model" is a property of theories. To show that it is a simple property we appeal to the Gregory-Ressayre theorem viz. If $T$ is a $\Sigma_{A}$ theory in $L_{A}$ for $A$ countable admissible, then $T$ has an uncountable model iff $T$ has a pair of models $M_{1}, M_{2}$ such that $M_{1} \prec \neq A M_{2}$.

The obvious candidate for the theory $T_{A}^{\prime}$ as described in Definition 1 is the following theory in the alphabet $L^{\prime}$ obtained by adding an additional unary predicate symbol $U$ to $L$ :

1. $\exists x \neg U(x)$,

2. $\varphi\left(x_{1}, \ldots, x_{n}\right) \rightarrow \varphi^{(U)}\left(x_{1}, \ldots, x_{n}\right)$, for each formula $\varphi\left(x_{1}, \ldots, x_{n}\right) \in$ $L_{A}$, and $n \in \omega$,

where $\varphi^{(U)}$ is the relativization of $\varphi$ to the predicate symbol $U . T_{A}^{\prime}$ is easily seen to give rise to an assignment of $\boldsymbol{\Sigma}$ theories in $L^{\prime}$. It only remains to verify (ii) of Definition 1.

This was essentially already done in [3], but we briefly sketch it again. Using the hypothesis of (ii) together with Lemmas 1 and 2, it follows that $M$ can be properly $L_{A}$ elementarily embedded in itself. However, using Lemma 1 it can be shown that the embedding is actually $L_{\infty \omega}$ elementary, which insures that the conclusion of (ii) holds.

AppliCation 2. We collect together a number of familiar properties, each of which can easily be seen to be a simple property of theories. In order to allow fixed parameters in the $\boldsymbol{\Sigma}$ theories given by an assignment, it sometimes becomes necessary to restrict our attention to those admissible sets containing the parameters. More significantly, the notion of simple property can be extended so as to allow structures for the original alphabet $\varrho$ to be relativized reducts of structures for $E^{\prime}$ rather than just reducts.

The existence of models with various automorphism properties, e.g. nonrigidity, infinitely many automorphisms, any element can be taken to any other element by an automorphism, an automorphism of order two, etc., are all easily seen to be simple properties of theories, as are the existence of models with elementary submodels or extensions of various strengths. Other notions will, no doubt, occur to the reader. 
One of these may be the existence of models with indiscernibles or ordered indiscernibles. The latter suggests that having models of arbitrarily large cardinality might be a simple property of theories. Here, however, one runs into difficulty in trying to find a structure generated from indiscernibles by Skolem functions. The analogue of Application 1 for arbitrarily large models is, as far as we know, open at this time.

3. The object of this section is to show that if a $\Sigma_{A}$ theory is sufficiently strong, then its $\boldsymbol{\Sigma}_{A}$-saturated models cannot be distinguished from one another on the basis of the simple properties of their $L_{\infty \omega}$ theories.

Definition 2. For any admissible $A$ a theory $T$ is said to be strongly complete $\boldsymbol{\Sigma}_{A}$ iff $T$ is $\boldsymbol{\Sigma}_{A}$ and for each sentence $\varphi$ of $L_{\infty \omega}$ of quantifier rank some ordinal of $A, T \vDash \varphi$ or $T \vDash \neg \varphi$.

The following corollaries to Theorem 1 are then immediate.

Corollary 1. Let $A$ be countable admissible and $T$ a strongly complete $\boldsymbol{\Sigma}_{A}$ theory, $M$ a $\boldsymbol{\Sigma}_{A}$-saturated model of $T$. Suppose $\mathrm{P}$ is a simple property of theories, then $T$ has property $\mathrm{P}$ iff $\mathrm{Th}_{\infty \omega}(M)$ has property $\mathrm{P}$.

Proof. One needs only note that $\mathrm{Th}_{M^{+}}(M)$ and $T$ are logically equivalent.

Corollary 2. Let $A, T$, and $\mathrm{P}$ be as above, with $M$ and $N \boldsymbol{\Sigma}_{A}$-saturated models of $T$. Then $\mathrm{Th}_{\infty \omega}(M)$ has property $\mathrm{P}$ iff $\mathrm{Th}_{\infty \omega}(N)$ has property $\mathrm{P}$.

We observe now that in the proof of Theorem 1 we only used the strength of the notion "property of theories" in the special case in which the theory is complete for $L_{\infty \omega}$. On the other hand, the proof of Corollary 1 seems to depend directly on the full strength of this notion. From the notion of being simple it does follow that if $T_{1}$ and $T_{2}$ are both primitive recursively definable on $A$ and $T_{2}$ is logically at least as strong as $T_{1}$, if $T_{2}$ has property $\mathrm{P}$, so does $T_{1}$. However, this does not seem to be sufficient for the proof of Corollary 1 .

In view of these observations, let us relax the notion "property of theories" and consider "weak properties of theories", for which one requires only that if $T_{1}$ and $T_{2}$ are logically equivalent $L_{\infty \omega}$ complete theories and $T_{1}$ has property $\mathrm{P}$, then $T_{2}$ does also. It is still possible to obtain a version of Corollary 2 , but not quite so easily.

Theorem 2. Let $A$ be countable admissible, $T$ a strongly complete $\boldsymbol{\Sigma}_{A}$ theory, and $M$ and $N \Sigma_{A}$-saturated models of $T$. Suppose $\mathrm{P}$ is a simple weak property of theories. Then $\mathrm{Th}_{\infty \omega}(M)$ has property $\mathrm{P}$ iff $\mathrm{Th}_{\infty \omega}(N)$ has property $\mathrm{P}$.

Proof. It is now useful to take $M$ and $N$ as models formed from urelements and assume $L$ to consist of pure sets. It is easy to see that this imposes no restriction on the isomorphism type of $M$ or $N$.

The key fact we need is that $\operatorname{HYP}(M)$ and $\operatorname{HYP}(N)$ have the same pure sets. This is shown in [4]. Since $T$ is strongly complete, it then follows that $\operatorname{Th}_{\mathrm{HYP}(M)}(M)=\operatorname{Th}_{\mathrm{HYP}(N)}(N)$, since all sentences in either theory have quantifier rank an ordinal of $A$. The result now follows directly from Theorem 1 which we noted held for weak properties of theories. 


\section{REFERENCES}

1. J. Gregory, Uncountable models and infinitary extensions, J. Symbolic Logic 38 (1973), 460-470.

2. M. Nadel, Scott sentences and admissible sets, Ann. Math. Logic 7 (1974), 267-294.

3. _ On models $\equiv_{\infty \omega}$ to an uncountable model, Proc. Amer. Math. Soc. 54 (1976), 307-310.

4. M. Nadel and J. Stavi, The pure part of hyp $(M)$, J. Symbolic Logic (to appear).

5. J.-P. Ressayre, Models with compactness properties relative to an admissible set (to appear).

Department of Mathematics, University of Notre Dame, Notre Dame, Indiana 46556 